\title{
Editorial
}

Psychotherapy and Psychosomatics
Psychother Psychosom 2013;82:353-354

DOI: $\underline{10.1159 / 000353599}$
Received: June 7, 2013

Accepted: June 10, 2013

Published online: September 20, 2013

\section{Benzodiazepines Revisited}

\author{
Richard Balon \\ Departments of Psychiatry and Behavioral Neurosciences and Anesthesiology, Wayne State University \\ School of Medicine, Detroit, Mich., USA
}

Since the arrival of chlordiazepoxide in 1959 and diazepam in 1960, benzodiazepines (BZPs) have been hesitantly lauded and frequently enthusiastically vilified by many. Nevertheless, new BZPs have been synthesized and by now, there are almost forty of them available around the world. This number alone is certainly a testimony to their wide use, usefulness and efficacy. Their anxiolytic properties are unquestionable. BZPs have been used in numerous clinical conditions and diagnoses - from all anxiety disorders (as defined by whatever diagnostic system) to sleep disorders, alcohol withdrawal and the augmentation of antidepressants and antipsychotics as well as for mania, muscle relaxation, seizures and in many other approved and nonapproved indications.

Interestingly, psychiatrists have been, at least in my experience, hesitant to use BZPs. This hesitancy has been reinforced by the emphasis on possible dependence, toxicity and abuse. Yet these issues have been overstated and overemphasized. In 1990, the American Psychiatric Association published a small monograph compiled by the American Psychiatric Association Task Force on Benzodiazepine Dependency, led by Carl Salzman [1]. The report focused specifically on the development of physiological dependence, especially at therapeutic doses, on acute and chronic toxicity and on the possible recreational use of BZPs as well as looking at the patterns of prescription and clinical use and on enabling psychiatrists to 'weight the relative benefits versus the risk of using ben- zodiazepines' and to develop some guidelines for the appropriate use of BZPs in clinical practice. The conclusion (too long to be cited in its entirety) contains a lot of interesting information. At that time, there were no data 'to suggest that long-term therapeutic use of benzodiazepines by patients commonly leads to dose escalation or to recreational abuse' [1, p. 55] (in all fairness, this was before the introduction of short half-life BZPs). The text stated that, at those times, a certain small percentage of patients used therapeutic doses of BZPs for self-medication of symptoms. The conclusion also discussed the development of physiological dependence, discontinuance symptoms and the relationship of the timing and severity of these to the half-life of the drug and the side effects of BZPs. The authors concluded that automobile driving is neither predictably nor consistently impaired by repeated therapeutic BZP doses. The report also noted that BZPs do not strongly reinforce their own use and are not widely abused drugs and that when abuse does occur, it is almost always among individuals who are also actively abusing alcohol, opiates or other sedative hypnotics $[1, \mathrm{p}$. 58]. The Task Force, after much cautious discussion and deliberation, came out in favor of careful prescribing of BZPs. The experts suggested that the benefits of BZPs clearly outweigh their hazards '(1) in patients with demonstrable persistent anxiety or dysphoric disorder or anxiety as a component of medical illness that cannot be otherwise treated and (2) in patients with chronic panic

\section{KARGER}

E-Mail karger@karger.com

www.karger.com/pps
C 2013 S. Karger AG, Basel

0033-3190/13/0826-0353\$38.00/0
Richard Balon, MD

Department of Psychiatry

Tolan Park Bldg 3rd Floor, 3901 Chrysler Service Dr.

Detroit, MI 48201 (USA)

E-Mail rbalon@wayne.edu 
or agoraphobic disorder when benzodiazepines are deemed the preferred pharmacological drug by the clinician' [1, p. 60].

Reviewing this carefully crafted report, the reader gets the message - BZPs are efficacious and clinically useful, yet they have some problems and should be prescribed judiciously. Well, this statement would probably fit any class of psychotropic medications. Nevertheless, BZPs are perceived as problematic for patients suffering from anxiety disorders such as panic or agoraphobia, and medications such as selective serotonin reuptake inhibitors (SSRIs) are promoted as being safer and more efficacious (although in some studies, the perceived lesser efficacy may just be the matter of the underdosing of BZPs). Now, Offidani et al. [2] and before them Berney et al. [3] present us with the sad message that the change in prescribing pattern favoring newer antidepressants over BZPs in the treatment of anxiety occurred without supporting evidence! Offidani et al. [2] also write that in trials comparing BZPs with newer antidepressants, BZPs were more efficacious and had fewer adverse effects in patients suffering from generalized anxiety disorder or panic disorder.

One wonders why the shift of opinion and the shift in the use of BZPs happened in this era of evidence-based medicine, without proper evidence? The explanation, in my opinion, is probably mainly due to the masterful propaganda of the pharmaceutical industry. At the time of the introduction of SSRIs and other new antidepressants, very little was known about their side effects. This usually happens with new classes of medications, as the longterm effects are detected only with long-term use, while the studies needed for regulatory approval are shortterm. Would anyone believe that the frequency of sexual side effects associated with fluoxetine is just $1.8 \%$ as was noted at the time of approval of this drug? The 'advantage' of SSRIs has been frequently reinforced by mentioning their regulatory approval for numerous anxiety disorder indications. BZPs certainly cannot match SSRIs in terms of number of regulatory approvals for various indications. Pharmaceutical companies no longer own the patent for BZP use, and thus approval in the indication(s) for a new anxiety disorder may not make business-sense (aside from the fact that some BZPs were introduced prior to the introduction of some of the diagnostic entities for which they are now used). Last but not least, psychiatrists are notoriously overcautious and tend to avoid using medications that may be considered prone to abuse or dependency or be 'difficult'. Thus, the issues of dependency, toxicity, abuse and the side effects of BZPs were probably overstated (perhaps even intentionally, although that would be hard to prove now) early in the era of promoting SSRIs. We did not know about the withdrawal symptoms associated with some of the SSRIs and other newer antidepressants, or their long-term side effects. I do not blame the industry, however, as much as I blame our profession for taking the seemingly easy road.

Medicine has been going through a time of 'invention crisis', a distressing decline in pharmaceutical innovation and an atrophy of serendipity [4]. One of the approaches to addressing this crisis is the reevaluation of the existing 'old' medications. Some disciplines, e.g. oncology, have done this already, examining old drugs in new indications, at times even outside of their main domain of treatment. For instance, imatinib was originally developed for chronic myelogenous leukemia, and is now used for other cancers. Bevacizumab, used originally for colon cancer and later for other cancers, is now being used for age-related macular degeneration. It appears to be high time for a similar soul-searching and scrutiny of treatment indications in psychiatry. We are probably not going to find totally novel or different indications for BZPs; however, we should definitely reconsider their use for anxiety disorders as well as reevaluate the hesitancy to use them in such indications.

\section{References}

1 The American Psychiatric Association Task Force on Benzodiazepine Dependency: Benzodiazepine dependence, toxicity, and abuse. Washington, American Psychiatric Association, 1990.

-2 Offidani E, Guidi J, Tomba E, Fava GA: Efficacy and tolerability of benzodiazepines versus antidepressants in anxiety disorders; a systematic review and meta-analysis. Psychother Psychosom 2013;82:355-362. $\checkmark 3$ Berney P, Halperin D, Tango R, DaenikerDayer I, Schulz P: A major change of prescribing pattern in absence of adequate evidence: benzodiazepines versus newer antidepressants in anxiety disorders. Psychopharmacol Bull 2008;41:39-47.

4 Klein DF: The loss of serendipity. JAMA 2008; 299:1063-1065. 